\title{
A Formative Study for Record-time Manual Annotation of First-person Videos
}

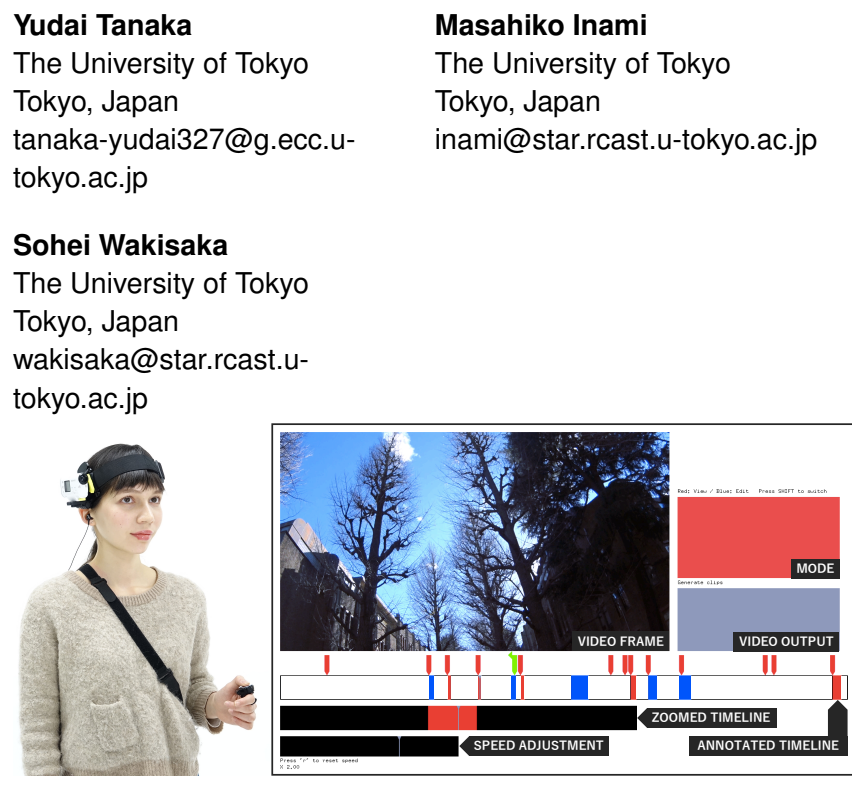

Figure 1: Left: Appearance of the wearable camera system. Right: The prototype video editing interface.

Permission to make digital or hard copies of part or all of this work for personal or classroom use is granted without fee provided that copies are not made or distributed for profit or commercial advantage and that copies bear this notice and the full citation on the first page. Copyrights for components of this work owned by others than ACM must be honored. Abstracting with credit is permitted. To copy otherwise, or republish, to post on servers or to redistribute to lists, requires prior specific permission and/or a fee. Request permissions from Permissions@acm.org.

MobileHCl'19 Adjunct, October 1-4, 2019, Taipei, Taiwan

() 2019 Association for Computing Machinery.

ACM ISBN $978-1-4503-6825-4 / 19 / 10$

\begin{abstract}
To efficiently edit first-person videos, manually highlighting important scenes while recording is helpful. However, little study has been performed on how such annotation contributes to video editing and affects user behavior during recording. To elicit fundamental requirements for designing useful record-time annotation techniques, we conducted a study using a set of prototype wearable camera system and a video editing interface that enables users to annotate scenes during recording. We asked participants to perform video recording and editing tasks with two different interface settings. We observed that the participants edited videos more efficiently with detailed annotation techniques, whereas focussing on annotating scenes affected their record-time behavior. We conclude the paper with the design guidelines developed from the findings.
\end{abstract}

\section{Author Keywords}

Video annotation; First-person videos; Context-aware video editing; Applications and experiences

\section{ACM Classification Keywords}

H.5.2 [Information Interfaces and Presentation (e.g., HCl)]:

User Interfaces: Interaction styles (e.g., commands, menus, forms, direct manipulation). 


\section{Introduction}

The increasing popularity of wearable cameras such as Gopro has resulted in the widespread practice of continuous video recording from a user's own perspective. Because videos emerging from such activity are often too lengthy for viewers to appreciate, it is necessary to apply summarization or efficient browsing techniques to original video materials. Various automatic video browsing and summarizing applications have been proposed hitherto. However, as such techniques are usually independent of a user's intention and personal preferences, they are sometimes inappropriate for video summarization where important scenes are decided by the user's subjective preference (e.g., favorite landscape, specific event, etc.). With important scenes manually highlighted during recording, in contrast, users can efficiently retrieve such scenes without missing. In spite of its usefulness, little research has been performed on how record-time manual annotation of egocentric videos facilitates context-aware video browsing.

In this study, we first present a prototype system for manual video annotation. The system consists of a wearable camera associated with a handheld controller interface and a video editing software. Users can adaptively highlight important scenes with the controller while recording a video. When they review the video, the highlight tags are displayed on a timeline of the video editing interface. Then, to investigate how record-time annotation techniques facilitate efficient video browsing and editing, we conducted a user study with the system. Through the study, we elicited that i) with various annotation functions provided, users can adaptively utilize them, whereas ii) the multifunctional system makes users more focused on record-time annotation, affecting their behaviors while recording. Based on the findings, this paper offers design guidelines for record-time manual annotation interfaces of first-person videos.

\section{Related Work}

Video Summarization and Browsing Support

A variety of video summarization methods have been studied. One example is the method of selecting keyframes based on how objects in a video interact with a user [5]. Further, scene extraction techniques exist that use sensed data such as user's gaze [9]. For creating a summarized video that is relevant to user preference, a scene extraction method referring to preference queries that users input beforehand [7] is practical. Browsing support techniques are also well established. Pongnumkulet et al. presented a video fast-browsing method that presents salient shots at an intelligible speed and others at a quick speed based on content analysis [6]. Higuchi et al. further investigated such adaptive video fast-forwarding methods by proposing a video browsing interface called EgoScanning with an elastic timeline emerging from variable computer vision analysis based on user input for featured target parameters (e.g., people, hand) [3].

\section{Manual Annotation}

Although manual intervention for activity and context recognition with wearable devices is common [1, 4], video recording interfaces associated with manual annotation have not been well explored. As a research involving recordtime manual annotation, Suzuki et al. proposed a video watermarking tool called Annotone [8]. Using Annotone, users can embed various contextual information using highfrequency audio signals during video recording. Unlike this study, our focus is on investigating how users manually interact with record-time annotation techniques. Some off-the-shelf products enable users to highlight important scenes on a real-time basis. GoPro's HERO 7 [2] is a compact digital video camera with which users can add annotation tags while recording videos using a button on the camera or an accessory handheld controller. 


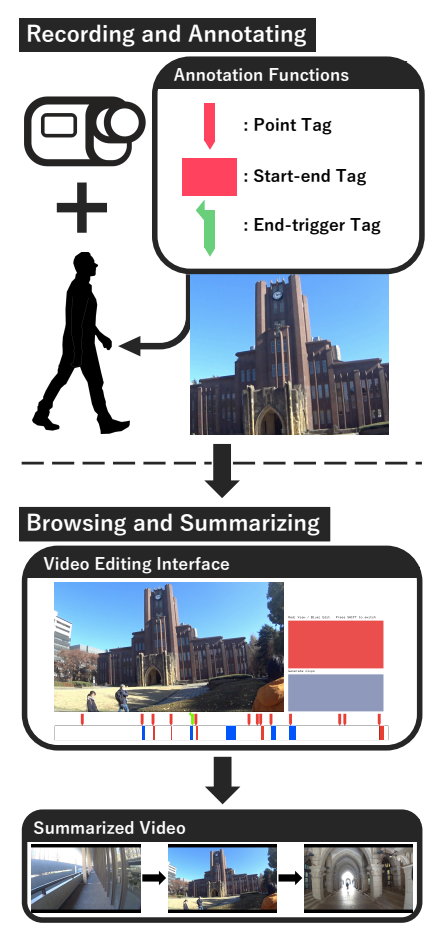

Figure 2: An outline of the video summarization process based on record-time manual annotation.

\section{System Design}

Assuming a scenario where a person records a video and later edits it, we designed a system that allows users to highlight scenes while recording to mitigate loads while editing (Figure 2). Using a controller, users set tags that are later displayed on the video timeline of the editing interface.

\section{Function}

We chose and implemented the following types of highlight tags, considering typical user demands while recording: i) concrete indication of scenes if possible and ii) annotating scenes without missing any important or memorable events.

Point tag: Users click a button to make a point tag appear on the editing interface (the red colored bookmark in Figure 2). It allows users to roughly notice where the important scenes are when they are browsing the video. This feature is the same as the GoPro's highlight function [2].

Start-end tag: By holding the button down for a certain duration and releasing it, users can highlight a specific range of time(the red colored belt in Figure 2). During browsing and editing, users can easily recognize the precise ranges of the important scenes and can instantly select clips for summarization by just clicking the tags.

End-trigger tag: Users click another button to create a tag that indicates a scene eight seconds prior to the moment when the button is triggered(the green bookmark in Figure $2)$. This tag is prepared for the cases in which users cannot determine or miss the scenes start (e.g., sudden appearances of interesting objects).

\section{Wearable Annotation Interface}

Users wear the equipments depicted in Figure 3, and the set up is shown in Figure 1 (Left): a video camera (SONY
HDR-AS100V) mounted on the head, the controller on the left forefinger, an earphone inserted to the right ear and a microcomputer (RaspBerry Pi3 Model $\mathrm{B}+$ ) in the shoulder bag. With the finger-wearable controller (Sanwa Wireless Ring Mouse 2), users can push the buttons for tagging without looking at the device. The middle button on the controller is responsible for point tag and start-end tag (Figure 3 ); clicking to set the former and holding down through the scene to set the latter. Clicking the right button activates end-trigger tag. Pushing any of the buttons, a user hears a beep sound corresponding to each type of tags through the earphone and notices that a tag is set correctly. The microcomputer connected to the controller records timestamps of tags on a real-time basis. After recording, a list of timestamps is exported to a laptop for browsing so that the tags appear in the editing interface later.

Browsing and editing interface

A user manipulates the editing interface(Figure 1 (Right)) with a mouse and a keyboard on a laptop. The playback speed can be changed with the maximum of $3 \mathrm{X}$ by adjusting the speed bar at the bottom. By clicking the annotated timeline, the user can move the playback points. When a user clicks a tag on the timeline, the player jumps to the start point of a highlighted scene (note: in the case of endtrigger tag, the viewer moves to eight seconds prior to the tag location). To summarize the video, a user selects clips by clicking the start point and the end point of scenes on the video timeline while pressing the SHIFT key. Pressing the key alters the color of the square window (upper-right in Figure 1 (Right)) from red to blue, which notifies users that clip selection is ready. A user can also but more accurately select the playback points and clips to be summarized by clicking the zoomed timeline displayed below the annotated timeline. After clip selection, one can push the video output button (middle-right in Figure 1 (Right)) to combine the clips 
and create a summarized video.

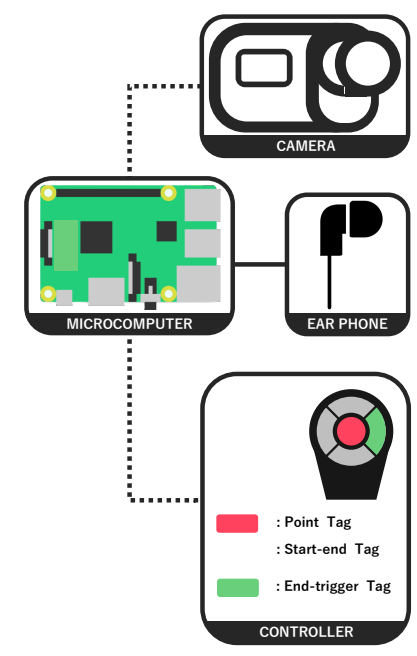

Figure 3: A diagram of the wearable system for record-time manual annotation: the solid line represent wired connection, the dotted lines represent wireless connection.

\section{User Study} additional loads to users.

\section{Conditions of Experiments}

We conducted an experiment to investigate how users interact with record-time video annotation techniques. Our hypothesis is that with multiple annotation functions, users can more easily browse and edit lengthy first-person videos, whereas focusing on annotation during recording becomes

Eight participants were recruited from the authors' university (7 students (male) and a postdoc (female); aged between 21 and 33). We compared the proposed interface described above with a baseline interface that only impelented point tag. All widgets other than the variety of tags remained the same in both conditions. We asked participants to make a short introduction movie of the university, assuming a typical situation where a person records and edits an egocentric video. We designed tasks in which participants actually record videos while walking in the university campus. We prepared two predetermined courses, A and $B$, such that one participant can experience both of the conditions. Participants followed the routes anti-clockwise with an experimental assistant. We carefully selected two courses so that both are equally interesting to typical sightseeners. The average walking time difference was negligible (A: 833 (std: 64.2), B: 841 (std: 94.4) sec).

\section{Procedure}

At the beginning, we gave participants a brief explanation of the study aim and the tasks. The session proceeded as follows: with the baseline interface, the participants left the venue where the remaining part of the study conducted and started walking one course. After they finished recording and returned to the venue, they were asked to edit the video that they had just recorded without time limit; we let them edit the video until they got satisfied with selected clips. Subsequently, they filled out a questionnaire about the interface usability. Then participants walked the other course, using the proposed interface, performed the tasks and answered questions in the same way. Finally, we conducted an exit interview. Before each recording and editing task, we gave the participants an instruction on the interface that they were about to use. The order of the courses was alternated, whereas the order of the interfaces was fixed to the baseline to the proposed. We intended to avoid participants' confusion when they first use the more complicated interface. We measured video editing time in each condition and utilization ratio of functions in the proposed condition. Each session took approximately two hours.

\section{Results}

Task Completion Time and Utilization of Functions

Figure 4 (Upper) shows the result of editing completion time for each participant. The proposed interface achieved faster task completion in all cases (note: the result can be biased by the order effect of interfaces). As shown in the utilization ratio of tags in Figure 4 (Middle), all participants used startend tag more than any other tag when using the proposed. Totally, in the proposed condition, more than $70 \%$ of annotation tags were from the functions not possessed by the baseline. Figure 4 (Lower) shows the details of how participants selected clips while editing in the proposed condition. There are four methods for selecting clips: i) apply ranges indicated by start-end tags (direct), ii) adjust the scopes of start-end tags (adjusted), iii) set scopes by reviewing scenes highlighted by point or end-trigger tag (point), and iv) select clip ranges independent of tags(no tag). All participants selected the clips based on start-end tag (direct, adjusted) in more than half of all cases. In total, more than $60 \%$ of the selected clips were from start-end tag. 


\begin{tabular}{|c|c|c|c|c|}
\hline & \multicolumn{2}{|r|}{ Baseline(B) } & Proposed(P) & \\
\hline A & $\bullet \rightarrow \bullet$ & 605 & 306 & \\
\hline B & $\bullet \rightarrow \bullet$ & 533 & 297 & 1.79 \\
\hline c & $\rightarrow$ & 446 & 284 & 1.7 \\
\hline D & $\bullet \rightarrow \bullet$ & 603 & 429 & 1.41 \\
\hline E & $\bullet \rightarrow$ & 536 & 443 & 1.21 \\
\hline $\mathrm{F}$ & $\rightarrow \bullet$ & 394 & 362 & 1.09 \\
\hline G & $\bullet \bullet$ & 365 & 355 & 1.03 \\
\hline $\mathrm{H}$ & $\bullet \rightarrow$ & 431 & 429 & 1.01 \\
\hline & $\begin{array}{l}\text { erage } \\
\text { Std }\end{array}$ & $\begin{array}{c}489.13 \\
92.74\end{array}$ & $\begin{array}{c}363.13 \\
64.39\end{array}$ & 1.35 \\
\hline
\end{tabular}

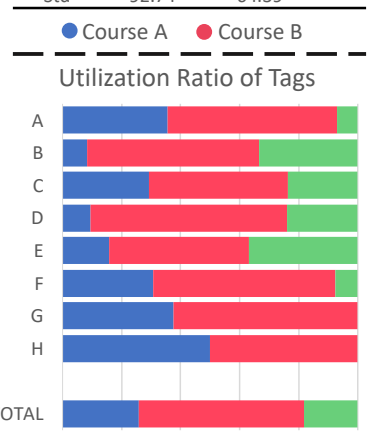

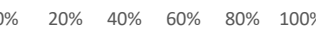
m Point $\amalg$ Start-end $\square$ End-trigger Clip Selection Methods

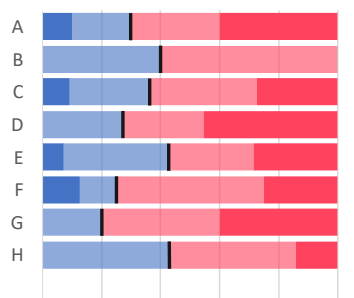

TOTAL $\begin{array}{llllll}0 \% & 20 \% & 40 \% & 60 \% & 80 \% & 100 \%\end{array}$ $\square$ No tag $\square$ Point $\square$ Adjusted $\square$ Direct

Figure 4: Upper: Comparison of summarization completion time [sec]. Middle: Utilization ratio of tags. Lower: Utilization ratio of clip selection methods.

\section{Questionnaire}

Participants filled out eight questions with 7-point Likert items (Figure 5). Apparently there was no significant difference in the first five questions for the usability comparison. One likely reason is the ceiling effect; the scores of the baseline is already high. Start-end tag and one-click clip selection were evaluated as valuable (EQ1 and EQ2), whereas there was considerable variation between individuals in the end-tigger tag leverage (EQ3) .

\section{Feedback and Observation}

At the end of the experiment, we conducted a $10 \mathrm{~min}$ semistructured interview. For the recording part, we asked "How did you use the interface to annotate scenes? Please answer for each condition," then "Did you feel any difficulty in using the interfaces?" and "Did you feel any changes when using different interfaces?" For the editing part, we asked "How did you browse the video and select the clips for summarization? Please answer for each condition," then "Did you feel any difference in editing videos using different interfaces?" Finally, we asked the participants any events they considered worth mentioning during the study.

With the interview, we found that participants mainly used start-end tag when highlighting scenes whose end timing were foreseeable (e.g., walking up to the historic auditorium then passing by) as indicated by comments such as "I used start-end tag when I could easily predict the button release timing." In the proposed condition, participants tended to use point tag when they did not define the certain ranges of scenes (e.g., "I utilized the function when I wanted to indicate some scenes vaguely"). End-trigger tag was applied in expected situations (e.g., "I tried to capture the moment of a crow had suddenly flown away by using the function").

In editing, participants utilized start-end tags on the video timeline to select clips. Most of the users mentioned about the usefulness (e.g., "As selecting clips by just clicking the start-end tags was really convenient, I mostly used the method"). Most of the participants mentioned that the proposed annotation functions made video summarization easier (e.g., "Since there was more information on the video timeline because of the multiple types of tags, I could easily understand what I wanted to highlight").

We elicited two types of participants' record-time behavior change with the proposed interface. One type is that they were more concentrating on annotating scenes (e.g, "I thought start-end tag was significantly useful to efficiently edit the video, so I was focused on using the cue during recording"). The other is that they were more aware of head movement and the camera angle while annotating scenes (e.g., "Using the start-end function, I tried to adjust and stabilize my head angle so that the object would be within the camera view"). Both changes are possibly attributed to that start-end tag enabled users to edit scenes while recording.

\section{Design Guidelines}

System designers can facilitate a user's apposite annotation by providing a set of annotation cues that is appropriate for the recording situations. A lesson from the start-end tag usage is that with a more concrete method of specifying the ranges of important scenes, the users can utilize it adaptively for efficient video editing. Further, we observed that some participants applied the end-trigger cue in the specific situations where the function is expected to be used. The findings suggest that by providing space for users to decide how to annotate certain scenes, they can use different methods appropriately for different situations, thus achieving efficient video editing.

Record-time annotation generally involves users in decision making which is usually performed during editing, imposing cognitive loads on them. Further, we observed some 


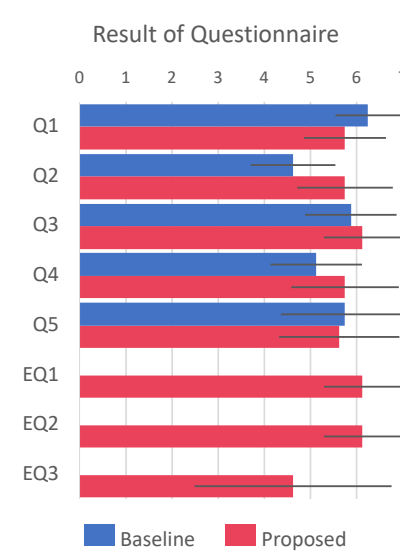

Q1: Ease of Using Controller

Q2: Appositeness of Annotation

Q3: Ease of Browsing

Q4: Ease of Editing

Q5: Ease of Retrieving

EQ1: Usefulness of Start-end Tag

EQ2: Usefulness of One-click Selection

EQ3: Usefulness of End-trigger Tag

Figure 5: Results of the questionnaire. The EQs were only asked after the task with the proposed interface. participants had changed the manner of head moving and attempted to stabilize the camera in the proposed condition. We should be aware of such users' behavioral change in addition to cognitive loads due to record-time annotation. Those behavioral states could be advantages for recording first-person videos in some situations. For instance, in video surveillance, introducing record-time editing functions may lead to the stabilization of a camera wearer's head and thus make videos less shaky.

\section{Conclusion}

In this study, we implemented a prototype system for manual video annotation that consists of a handheld controller and a video editing software. We conducted a user experiment as a first step to investigate how such record-time annotation techniques facilitate efficient video editing and affect user's behavior while recording videos. The design guidelines based on the findings from the user study will contribute to better development of record-time manual annotation interfaces for first-person videos.

\section{REFERENCES}

1. David Bannach, Kai Kunze, Jens Weppner, and Paul Lukowicz. 2010. Integrated tool chain for recording and handling large, multimodal context recognition data sets. In Proceedings of the 12th ACM international conference adjunct papers on Ubiquitous computing-Adjunct. ACM, 357-358.

2. GoPro. 2014. What is HiLight Tagging \& How Does it Work? (2014). Retrieved January 29, 2019 from https : //gopro.com/help/articles/Question_Answer/ What-is-HiLight-Tagging-and-How-Does-it-Work

3. Keita Higuchi, Ryo Yonetani, and Yoichi Sato. 2017. EgoScanning: quickly scanning first-person videos with egocentric elastic timelines. In Proceedings of the 2017
CHI Conference on Human Factors in Computing Systems. ACM, 6536-6546.

4. Shoya Ishimaru, Andreas Poxrucker, Paul Lukowicz, Jens Weppner, Kai Kunze, and Koichi Kise. 2014. Shiny-an activity logging platform for Google Glass. In 2014 ACM International Joint Conference on Pervasive and Ubiquitous Computing, UbiComp 2014.

Association for Computing Machinery, Inc, 283-286.

5. Yong Jae Lee, Joydeep Ghosh, and Kristen Grauman. 2012. Discovering important people and objects for egocentric video summarization. In Computer Vision and Pattern Recognition (CVPR), 2012 IEEE Conference on. IEEE, 1346-1353.

6. Suporn Pongnumkul, Jue Wang, Gonzalo Ramos, and Michael Cohen. 2010. Content-aware dynamic timeline for video browsing. In Proceedings of the 23nd annual ACM symposium on User interface software and technology. ACM, 139-142.

7. Aidean Sharghi, Boqing Gong, and Mubarak Shah. 2016. Query-focused extractive video summarization. In European Conference on Computer Vision. Springer, 3-19.

8. Ryohei Suzuki, Daisuke Sakamoto, and Takeo Igarashi. 2015. AnnoTone: Record-time audio watermarking for context-aware video editing. In Proceedings of the 33rd Annual ACM Conference on Human Factors in Computing Systems. ACM, 57-66.

9. Jia Xu, Lopamudra Mukherjee, Yin Li, Jamieson Warner, James M Rehg, and Vikas Singh. 2015. Gaze-enabled egocentric video summarization via constrained submodular maximization. In Proceedings of the IEEE Conference on Computer Vision and Pattern Recognition. 2235-2244. 